\title{
Digitale Aktionsraumforschung. Neue Methoden zur Analyse von Bewegungsprofilen im städtischen Raum
}

\section{Social-Movement Pattern Analysis: New Digital Methods to Analyse Activity Space in Urban Areas}

https://doi.org/10.2478/rara-2019-0027

Eingegangen: 20. Juni 2018; Angenommen: 28. Februar 2019

\begin{abstract}
Zusammenfassung: Der in diesem Beitrag vorgestellte Forschungsansatz soll als Vorschlag für eine Neuauflage der stadtsoziologischen und stadtgeographischen Aktionsraumforschung verstanden werden. Der methodische Ansatz wird dabei anhand einer explorativen Studie mit Koblenzer Studierenden veranschaulicht. Entsprechend dem Mixed-Methods-Ansatz „Explanatory Sequential Design“ kombinieren wir quantitative und qualitative Erhebungs- und Auswertungsverfahren. So erheben wir studentische Bewegungsdaten mithilfe einer Smartphone-App, um studentische Hotspots zu lokalisieren. Zudem verknüpfen wir diese Daten mit Angaben zu den individuellen Lebensstilen der Studenten, um dadurch eine Differenzierung der Bewegungsabläufe zu erreichen (Bewegungsmuster). Schließlich ergänzen wir die erhobenen Daten mit qualitativen Beobachtungen der lokalisierten Hotspots, um ein besseres Verständnis für die Gründe der Bewegungsmuster zu entwickeln. Ohne Anspruch auf Repräsentativität zu erheben, zeigen unsere Ergebnisse, dass sich die Bewegungsprofile bzw. die identifizierten Lokalitäten entlang der lebensstilspezifischen Differenzierungskriterien beschreiben lassen. Werden die jeweiligen Orte mittels qualitativer Beobachtungen näher betrachtet, dann lassen sich Anzeichen einer engen Passung von theoretisch implizierten Annahmen zu den Lebensführungstypen und den Raumbezügen einerseits und den aufgesuchten Lokalitäten andererseits feststellen.
\end{abstract}

Schlüsselwörter: Aktionsraumforschung, Bewegungsprofile, Mixed Methods, GPS-Tracking, Lebensstil

\begin{abstract}
In this paper, we introduce a research approach which wants to be understood as a proposal for a new version of urban sociological and geographical activity-space research. The methodological approach is illustrated by an explorative study with students from Koblenz (Germany). According to the mixed-methods approach "Explanatory Sequential Design", we combine quantitative and qualitative survey and evaluation procedures. Thereby, we collect student movement data with the help of a smartphone app in order to localise student "hotspots". Additionally, we link them to information about the students' individual lifestyles so as to achieve a differentiation of their movement sequences (movement patterns). Finally, we complement the collected data with qualitative observations of the localised "hotspots" in order to develop a closer understanding of the "Why?" of the movement patterns. Without

\footnotetext{
*Corresponding author: Dr. Marc Hannappel, Universität Koblenz-Landau, Institut für Soziologie, Universitätsstraße 1, 56070 Koblenz, Deutschland, E-mail: marchannappel@uni-koblenz.de

Philipp Jakobs: Rheinische Friedrich-Wilhelms-Universität Bonn, Institut für Politische Wissenschaft und Soziologie, Lennéstraße 25, 53113 Bonn, Deutschland
} 
raising a claim for representativeness, our results show that the movement patterns and identified localities can be described along lifestyle-specific differentiation criteria. When the respective locations are regarded more closely, indications for a close fit between the theoretically implicated assumptions regarding lifestyle types and spatial relationships on the one hand and the sought out localities on the other hand can be identified.

Keywords: Activity space research, Movement patterns, Mixed methods, GPS-tracking, Lifestyle

\section{Einleitung}

In der soziologischen Stadtforschung besteht weitestgehend Einigkeit darüber, dass sich die Wohnstandorte von Angehörigen verschiedener gesellschaftlicher Gruppierungen meist nicht gleichmäßig über ein Stadtgebiet verteilen (vgl. z. B. Cutler/Glaeser/Vigdor 1999; Farwick 2001; Häußermann/Siebel 2004; Farwick 2012; Dangschat 2014). Vielmehr entstehen oftmals Stadtviertel oder Quartiere mit besonders hoher räumlicher Konzentration bestimmter (soziokultureller) Bevölkerungsgruppen. So kommen beispielsweise Helbig und Jähnen in einer aktuellen Studie zu dem Ergebnis, dass die soziale Segregation seit 2005 sogar zugenommen hat - ein Prozess, der in ähnlicher Form seit Mitte der 1990erJahre zu beobachten ist (Helbig/Jähnen 2018: 28). Solche residentiellen Segregationsprozesse werden immer dann problematisch, wenn sie in Armutsquartieren zur Verstetigung von Benachteiligung der Bewohner und somit zur Verfestigung sozialer Ungleichheit beitragen (Farwick 2001: 176 ff.; ILS/Strohmeier 2003: 3; Siebel 2012: 468 f.).

In ähnlicher Form herrscht auch in kultursoziologischen Diskursen nahezu Einigkeit darüber, dass sich Personen in der Art und Weise, wie sie ihren Lebensalltag bestreiten, nach soziokulturellen Kriterien beschreiben lassen. So zeigen verschiedene Studien Unterschiede in Bezug auf die individuelle Freizeitgestaltung auf (z. B. Vester 2015). Personen und Personengruppen lassen sich beispielsweise nach Maßgabe der von innen aufgesuchten Kultureinrichtungen (Reuband 2018), nach ihrem Konsumverhalten und Geschmack (Bourdieu 1982; Blasius/Winkler 1989) oder nach übergreifenden Konzepten wie Lebensstilen oder Milieus differenzieren (Schulze 1992; Schneider/Spellerberg 1999; Klee 2001; Otte 2004).

Wenn sich Menschen in Abhängigkeit von ihrer sozialen Lage zunehmend an verschiedenen (städtischen) Orten niederlassen, dann zeigt sich das in den segregierten Strukturen der Wohngebiete. Wenn sie aber zudem in unterschiedlichen Geschäften einkaufen, wenn sie an unterschiedlichen Orten ihre Freizeit verbringen und zusätzlich ihre Kinder auf verschiedene Schulen schicken, dann sollten sich auch Differenzen in ihrem Bewegungsverhalten jenseits der Wohngebiete zeigen. Während zu den benachteiligenden Effekten segregierter Wohngebiete sowie den exkludierenden Distinktionsprozessen in Bezug auf kulturelle Verhaltensweisen schon zahlreiche Studien vorliegen, ist mit den Differenzen der Bewegungsmuster ein Faktor gekennzeichnet, der bisher noch kaum erforscht worden ist und dementsprechend auch in stadtpolitischen Entscheidungen bisher kaum Beachtung finden konnte. Diese ,mobilen Segregationsmuster', das heißt, die Strukturen der räumlichen Ungleichheit jenseits des Wohngebietes zu erheben und der Stadtplanung und Stadtpolitik als handlungsleitendes Wissen zur Verfügung zu stellen, ist das Ziel unseres Beitrags.

Differenzierungen in den von Personen aufgesuchten Orten, die jenseits des Wohngebietes liegen, können mit den Methoden der herkömmlichen sozialen Segregationsforschung (z. B. Fragebogenerhebungen, amtliche Städtestatistik, qualitative Beobachtungen) nicht oder nur sehr aufwendig erfasst werden. Eine mögliche Annäherung an solche Differenzierungsprozesse besteht in den Methoden der Aktionsraumforschung. Diese fragt nach den individuellen Bewegungsmustern der Stadtbewohner im Stadtgebiet, welche sich auf der anderen Seite als besuchte Orte in der Gelegenheitsstruktur der Stadt darstellen (Dangschat/Droth/Friedrichs et al. 1982; Friedrichs 2011). Während die Aktionsraumforschung aber auf retrospektive Verfahren wie reflexive Interviews oder Tagebücher zurückgreifen musste, um die realen Bewegungsabläufe erfassen zu können, stehen hierfür heute moderne Technologien, wie GPS-Tracking und Ortungsdienste auf Smartphones zur Verfügung, welche die tatsächlichen physisch-geographischen Standpunkte aufzeichnen können. Konzeptionell kann unser Vorhaben insofern auch als Vorschlag für eine Erneuerung der Aktionsraumforschung gelesen werden.

Während solche digitalen Erhebungen in gewinnorientierten Unternehmen bereits seit längerem durchgeführt werden, fehlen bislang einschlägig soziologische Ansätze, die über die reine Erhebung und konsumspezi- 
fische Auswertung hinaus objektive Erkenntnisse bereitstellen und diese in ihrer gesellschaftlichen Relevanz adäquat einordnen können. Entsprechend diesem Desiderat verfolgt unser Ansatz eine dreifache Fragestellung:

- Lassen sich Bewegungsprofile entlang soziokultureller Merkmale beschreiben?

- Lassen sich städtische Orte identifizieren, die sich in Bezug auf soziokulturelle Merkmale inrer Nutzer durch eine besonders heterogene oder besonders homogene Nutzerstruktur auszeichnen?

- Durch welche inhaltlichen Merkmale zeichnen sich die jeweiligen Orte als erfahrbare Qualitäten aus?

Während Ersteres eine methodische Frage ist bzw. sich auf die Umsetzbarkeit des Vorhabens bezieht, enthalten die Punkte zwei und drei auch eine gesellschaftspolitische Dimension, die auf die Bedeutung von mobilen Segregationsprozessen für gesellschaftliche Differenzierungsprozesse verweist. Gordon W. Allport hat bereits in seinem erstmals 1954 erschienenen Werk "The nature of prejudice" auf die Bedeutung von zwischenmenschlichen Kontakten und Begegnungen für die Bildung von Vorurteilen hingewiesen (Allport 1971). Pettigrew und Tropp (2006) konnten mit einer umfangreichen Metastudie 50 Jahre später nachweisen, dass sich der Zusammenhang zwischen Intergruppenkontakten und der Bildung von Vorurteilen auch jenseits ethnischer Gruppen zeigen lässt. Begegnungen von Menschen unterschiedlicher ethnischer und sozialer Gruppen können nach dieser Lesart eine wichtige Voraussetzung für den Abbau von Vorurteilen zwischen diesen Gruppen beitragen. Die Funktion, Menschen zusammenzubringen, erfüllten in der Vergangenheit unter anderem öffentliche Räume. So weist Klamt (2012: 789) auf die Bedeutung (physischer) öffentlicher Räume für Demokratie, Diskurs und Liberalität hin. Obwohl die demokratietheoretische Bedeutung öffentlicher Räume kaum bestritten wird, ${ }^{1}$ wurden gerade in der zweiten Hälfte des 20. Jahrhunderts öffentliche Plätze derart umgestaltet, dass sie diese Funktion nicht mehr erfüllen konnten. Sennett (2001: 28) spricht hier sogar von einer „Zerstörung von lebendigem öffentlichen Raum", die durch einen Funktionswandel der Räume eingeleitet wurde. Maxime der Raumgestaltung öffentlicher Plätze war nicht mehr die, Menschen zum Verweilen anzuregen und so Möglichkeiten der Begegnung zu

1 Auf der anderen Seite sollte die Bedeutung öffentlicher Räume auch nicht überschätzt werden. „Given the complexity and adaptability of human species it would be far too simplistic to say that the way we design the urban realm has a direct influence on how everyone will behave in it" (Shaftoe 2008: 31). schaffen, sondern eine möglichst schnelle Durchquerung des Raums zu ermöglichen, um auf kürzestem Wege innerstädtische Ziele wie Einkaufszentren, Ärzte und Schulen zu erreichen. Dies führte nach Sennett (2009: 14) zu einer „Verödung und Trivialisierung der Stadt als Schauplatz des Lebens".

Die Analyse urbaner Räume bzw. die Suche nach diesen ist somit ein wichtiger Gegenstand soziologischer und humangeographischer Forschungen. Durch neue technische Innovationen der Datengewinnung und Datenanalyse sowie neue Möglichkeiten der Methodenkombination lassen derartige Untersuchungen neue Einblicke in gesellschaftliche Differenzierungs- und Segregationsprozesse erwarten.

Hierfür werden im Sinne eines Mixed-Methods-Konzeptes neben quantitativen Auswertungen der Bewegungsdaten auch qualitative Verfahren der teilnehmenden Beobachtung miteingeschlossen. Kurz gesagt: Die quantitativen Daten dienen der Lokalisation und Einordnung häufig frequentierter Orte, den sogenannten Hotspots. Mithilfe der qualitativen Beobachtungen sollen zusätzlich die Hintergründe über die Nutzungsmotive ermittelt werden, um ein tiefergehendes Verständnis für das Aufsuchen oder das Fernbleiben bestimmter Bevölkerungsgruppen von bestimmten städtischen Orten bzw. Gelegenheiten zu erhalten.

Die Analysen beschränken sich in diesem Beitrag auf die Erfassung und Auswertung studentischer ${ }^{2}$ Bewegungsprofile und somit auch auf die Analyse der von Studierenden aufgesuchten Orte. Die in Kapitel vier vorgestellten Ergebnisse sind aufgrund der kleinen Stichprobe nicht repräsentativ. Im Vordergrund des Beitrages stehen weniger die Ergebnisse als vielmehr die methodische Konzeption unseres Forschungsdesigns, also die Beschreibung der Entwicklung und Durchführung eines neuen Erhebungs- und Auswertungskonzeptes, das prinzipiell auf viele weitere Personengruppen und Forschungsfelder (z. B. Analyse von Gentrifizierungsprozessen, soziale Segregation der Bevölkerung) ausgeweitet werden kann. Die Ergebnisse demonstrieren aber sehr deutlich, welche Potenziale mit derartigen Ansätzen, die moderne Formen der Datengewinnung (GPSTracking via Smartphone) mit traditionellen Formen der Befragung (standardisierter Fragebogen) und qualitati-

2 Die Beschränkung auf studentische Bewegungsprofile ist darin begründet, dass eine flächendeckende Erhebung in der Bevölkerung einen enormen logistischen und finanziellen Aufwand bedeutet hätte. Ferner ist der Zugang zu Studierenden allein institutionell bedingt einfacher und es lassen sich kostengünstigere Anreize für eine Beteiligung setzen. 
ven Beobachtungen (qualitative Beschreibung der Lokalitäten) miteinander kombinieren, verbunden sind.

Im Folgenden werden wir zunächst unseren Forschungsansatz im Kontext der stadtsoziologischen und stadtgeographischen Aktionsraumforschung verorten und inn als eine sinnvolle methodische Erneuerung derselben formulieren (Kapitel 2). Daraufhin werden wir tiefgehender auf unsere Erhebungsmethode eingehen und herausarbeiten, welche praktischen Probleme im Zuge empirischer Forschungen auftreten können (Kapitel 3). Im darauf folgenden Kapitel 4 soll dann anhand unserer eigenen Studie exemplarisch vorgeführt werden, wie die Erhebung und Auswertung von digitalen Bewegungsprofilen in praxi aussehen könnte. Kapitel 5 enthält das Fazit.

\section{Aktionsraum}

Während die Aktionsraumforschung in den 1970er- und 1980er-Jahren ein wichtiges Forschungskonzept für die Stadtanalyse und Stadtforschung war, geriet sie in den letzten Jahrzehnten etwas in Vergessenheit (Hesse 2011: 25 ff.). Die Arbeit von Kühl und Wörmer (2012) über „Aktionsräume in polyzentrischen Stadtregionen" bildet eine von wenigen Ausnahmen.

Die Aktionsraumforschung knüpft an die Überlegungen von Frank E. Horton und David R. Reynolds an, die in ihrer viel beachteten Studie „Effects of urban spatial structure on individual behavior" eine begriffliche Differenzierung zwischen „objective spatial structure“, „action space" und "activity space" vornahmen (Horton/Reynolds 1971: 37). Ins Deutsche übersetzt wurden die Begriffe etwas später durch Jürgen Friedrichs, der die Aktionsraumforschung in die hiesige Stadtforschung eingeführt und weiterentwickelt hat. Friedrichs (1983: 306) unterscheidet zwischen der objektiv räumlichen Stadtstruktur („objective spatial structure“), dem subjektiven Stadtplan (,action space“) und dem Aktionsraum (,activity space“). Die objektive Stadtstruktur gleicht am ehesten der Perspektive auf Stadt, die auch bei der Erstellung eines professionellen Stadtplanes eingenommen wird. Es handelt sich hierbei um eine Topographie, die ein geographisch abgrenzbares Gebiet umfasst, in der wichtige infrastrukturelle Merkmale wie Straßen- und Schienennetz, kulturelle Einrichtungen, Sehenswürdigkeiten, Krankenhäuser und anderes markiert und lokalisiert sind (Friedrichs 1990: 167). Der subjektive Stadtplan ist ein Ausschnitt aus der objektiven Stadtstruktur und meint ganz allgemein den "Teil der Stadt, den ein Bewohner kennt" (Friedrichs 1990: 168). Es handelt sich demnach um sub- jektive Wissensbestände über einen geographischen Raum und dessen Ausstattung (Friedrichs 1983: 310), die von den Stadtbewohnern angeeignet werden. Der subjektive Stadtplan wird vorrangig durch die Aktivität einer Person erschlossen. Dazu gehören alltägliche Tätigkeiten, wie der Weg zur Arbeitsstätte, zur Schule oder der Einkauf, aber auch außeralltägliche Erfahrungen wie ein Theater- oder Kinobesuch, das Schlendern in der Fußgängerzone, ein Spaziergang oder eine Schifffahrt auf dem Rhein. Andererseits gibt es viele Orte, Plätze oder ganz allgemein Gelegenheiten in der Stadt, in der man lebt, von deren Existenz man zwar weiß, die man aber noch nie oder zumindest noch nicht häufig aufgesucht hat. Auch Internetrecherchen über Google Maps oder Map.de gehören zum Aneignungsprozess subjektiver Stadtpläne. Im Gegensatz dazu bezeichnet der Aktionsraum "the subset of all urban locations with which the individual has direct contact as the result of day-to-day activities" (Horton/Reynolds 1971: 37). Folglich müssen Begegnungen stattgefunden haben, das heißt, die physische Präsenz an einem Ort ist eine Voraussetzung, um von einem Aktionsraum sprechen zu können. Zusätzlich müssen aber noch diese Begegnungen eine gewisse Regelmäßigkeit oder Alltäglichkeit aufweisen.

Der Begriff „Aktionsraum“ konzentriert sich demnach auf konkrete Orte, die im Rahmen alltäglicher Unternehmungen aufgesucht werden. Geographisch lassen sich diese Orte als Punkte auf einer Karte abbilden. Verbindet man diese Punkte, dann ergeben sich subjektive Bewegungsprofile. Die Aktionsraumforschung steht in enger Verbindung zu Ansätzen zur Analyse städtischer Segregationsprozesse und Kontexteffekte. Insofern knüpft sie an eine lange Tradition stadtsoziologischer Forschung an.

Auf Robert E. Park (1915) geht die Vorstellung zurück, dass im Zuge der Arbeitsteilung und den damit einhergehenden sozialen Differenzierungen auch räumliche Differenzierung (residentielle Segregation) entsteht, die letztlich zu „natural areas“ führt. „Natural areas“ sind nach Park städtische Gebiete mit einer relativ homogenen Wohnbevölkerung. „Natural“ sind sie, insofern die Segregationsprozesse nicht nach ordnungspolitischen Regeln verlaufen, also nicht intendiert sind, sondern weil sie naturähnlichen "Gesetzmäßigkeiten" folgen (Park 1952: 178). Diese Gesetzmäßigkeiten beziehen sich aber nicht auf wirkliche Naturphänomene, sondern auf hintergründige soziale Prozesse wie „ökonomische Konkurrenz, die Auswirkungen von Grundstücksspekulationen“ sowie auf „technische [...] und kulturelle [...] Faktoren für die städtischen und gesellschaftlichen Selektions- und Segregationsprozesse“ (Krämer-Badoni 1992: 23). 
Ähnlich bedeutsam wie „natural areas“ sind für Parks Stadtsoziologie „moral regions“ (Park 1915: 610). Während sich die "natural areas“ mehr aus gruppengebundenen, das heißt nicht frei gewählten, Interessen und Notwendigkeiten heraus bilden, entstehen darüber hinaus auch Regionen durch die Segregation von „Leidenschaften und Temperamente[n], die nicht so eindeutig an soziale Gruppen und ihre spezifischen Gebiete gebunden sind, die sich vielmehr um bestimmte Aktivitäten bilden" (Häußermann/Siebel 2004: 53). Neben einer Stadtstruktur, die durch sozialstrukturelle Merkmale bestimmt ist, entwickelt sich also eine zweite, mehr oder weniger kongruente Stadtstruktur, die durch das bestimmt ist, was heute zumeist mit dem Begriff „Lebensstil“ bezeichnet wird. Versteht man „natural areas" nicht nur als vollständig segregierte Gebiete und „moral regions" entsprechend nicht nur als einem einzelnen Milieu zugeordnete Opportunitätsstrukturen (vgl. Friedrichs 2011: 37 ff.), dann finden sich bereits bei Park zwei Grunddimensionen der Aktionsraumforschung, nämlich Wohnquartier und Gelegenheitsstruktur.

Mit dem Begriff der „natural areas“ wurde ein Grundstein für die heute noch zur Analyse gesellschaftlicher Strukturen im urbanen Raum verwendete Segregationsforschung gelegt. Viele Studien konzentrieren sich auf residentielle Segregationsprozesse, die beispielsweise nach dem Einkommen der Bewohner oder dem Anteil der Sozialhilfeempfänger in einem Stadtteil differenzieren (vgl. Farwick 2001; Blasius/Friedrichs/ Symann 2007), auf ethnische Segregation (z. B. Cutler/ Glaeser/Vigdor 1999) oder auf eine Überlagerung von beiden (vgl. Erdmann 2001; Friedrichs/Triemer 2009). Andere Studien erforschen aber auch die Segregation von Altersgruppen (z. B. Schütz 1985) oder von spezifisch operationalisierten Lebensformen (Eder Sandnter/ Schneider-Sliwa 2007).

Als sinnvolle Ergänzung von Studien zu residentiellen Segregationsprozessen und deren Kontexteinflüssen eignen sich Ansätze, die wie die Aktionsraumforschung die Aktivität einer Person im gesamten städtischen bzw. alltäglich genutzten Gebiet zum Gegenstand ihres Untersuchungsdesigns machen. Analog zur Segregationsforschung, die von der Annahme geleitet wird, dass sich die Wohnorte von Personen in Abhängigkeit sozialstruktureller Merkmale nicht gleich über ein Stadtgebiet verteilen, geht die Aktionsraumforschung davon aus, dass sich auch die Aktionen von Personen entlang sozialer Kriterien differenzieren lassen. Mit anderen Worten: Auch jenseits von segregierten Gebieten strukturieren sich die Bewegungsmuster und Aufenthaltsorte von Stadtbewohnern nicht zufällig, sondern in Abhängigkeit von Wahrnehmungs-, Deutungs- und Handlungsmustern, die zumeist mit spezifischen soziodemographischen Merkmalen korrelieren.

Ziel der Aktionsraumforschung ist „die Beschreibung und Erklärung raumbezogenen Verhaltens von Personen oder Mengen von Personen" (Dangschat/Droth/ Friedrichs et al. 1982: 4). Demzufolge steht nicht allein die Aktivität im Vordergrund des Forschungsinteresses, sondern auch die Orte, an denen bestimmte Tätigkeiten ausgeführt werden. Für die soziologische Forschung werden derartige Forschungsansätze aber erst dann relevant, wenn sie zu den ortsbezogenen Handlungen auch soziodemographische Individualmerkmale erheben (vgl. Friedrichs 2011: 36).

Individuelle Aktionsräume werden in der Regel mittels standardisierter Fragebögen oder durch die Auswertung extra für das Forschungsvorhaben angefertigter Tagebücher erhoben (vgl. Friedrichs 1983: 315). In beiden Fällen ist es wichtig, dass Ort, Zeit und Aktion erfasst werden. Zur Erhebung dieser „time-space budgets" (Weber/Bauder 2013: 101) werden Probanden entweder gebeten, Tagebuch über die an einem Tag durchgeführten Tätigkeiten zu führen oder sie werden retrospektiv danach befragt. So ermitteln beispielsweise Kühl und Wörmer (2012) individuelle Aktionsräume mittels einer schriftlichen Befragung, im Rahmen derer die Befragten elf verschiedene alltägliche Aktionen wie Lebensmitteleinkauf, Sport, Freunde treffen oder den Besuch von Ärzten, Friseur oder Musik- und Kulturveranstaltungen angeben sollten (vgl. Kühl/Wörmer 2012: 223). Eine etwas differenziertere Erfassung der individuellen Aktionsräume schlägt Friedrichs (1990) vor. Mithilfe sogenannter Raum-Zeit-Pfade sollen hier individuelle Tagesabläufe rekonstruiert werden.

Diese Art der Erhebung hat viele Nachteile. So wird beispielsweise bemängelt, dass die Forscher zur Erhebung der Aktionsräume bereits a priori entscheiden müssen, wie feingliedrig sie die zu erhebenden Aktionsräume und Gelegenheiten differenzieren wollen (Dangschat/Droth/Friedrichs et al. 1982: 31, 49; Friedrichs 1990: 164). Zudem stellt sich die Frage, welche Tätigkeiten zu den erhobenen Aktionsräumen hinzugerechnet werden sollen. Meist ist die Häufigkeit der Ausübung einer Aktion das ausschlaggebende Kriterium, aber wie groß diese sein muss, liegt im Ermessensspielraum der Forscher (Dangschat/Droth/Friedrichs et al. 1982: 31). Grundlegender als diese forschungspraktischen und letztlich kontingenten Entscheidungen ist allerdings die Angewiesenheit auf sozusagen ,subjektive' Instrumente zur Erhebung der ,objektiv' besuchten Orte. Die Erhebung über Tagebücher birgt die Gefahr, dass die Pro- 
banden sich nicht regelmäßig Notizen machen und das Tagebuch später auf einige Tage rückblickend ausfüllen (Dangschat/Droth/Friedrichs et al. 1982: 75). Auch die Interviewmethode bleibt abhängig von der Erinnerungsfähigkeit und der Erinnerungsbereitschaft der Interviewten (Dangschat/Droth/Friedrichs et al. 1982: 75f.).

Insbesondere da die so gewonnenen Aktionsräume als objektiv besuchte Orte den subjektiven Stadtplänen gegenübergestellt werden, scheint eine Basis aus subjektiven Erhebungsmethoden unsicher. Verzerrungen zugunsten der auch im subjektiven Stadtplan erfassten, im aktiven Gedächtnis vorhandenen Orte sind wahrscheinlich. Diese Problematik konnte die Aktionsraumforschung weitestgehend neutralisieren, indem sie den Aktionsraum eines Stadtbewohners als Teilausschnitt seines subjektiven Stadtplans wertet. Aus unserer Sicht scheint es aber durchaus möglich, dass sich Orte im Aktionsraum finden, die sich zumindest nicht im bewussten oder aktiven subjektiven Stadtplan widerspiegeln.

Zusammenfassend lassen sich vier zentrale Problembereiche der Aktionsraumforschung kennzeichnen: (1) Selektions- und Klassifikationsprobleme, also Probleme der Auswahl und Gliederung von Aktionen und Gelegenheiten, (2) Operationalisierungsprobleme, also solche der methodischen Umsetzung und Messbarkeit von Aktionsräumen, und (3) Erhebungsprobleme, welche vor allem auf die Subjektivität und Selektivität der vorhandenen Instrumente zurückzuführen sind. (4) Selbst wenn diese Probleme gelöst würden, fehlt immer noch eine Erklärung, weshalb bestimmte Orte von bestimmten Gruppen aufgesucht werden und andere nicht.

Um diesen Problemen zu begegnen, kann heute auf moderne Methoden der Datenerfassung und Auswertung zurückgegriffen werden. Für die Aktionsraumforschung bieten sich Erhebungsmethoden an, die räumliche Bewegungen von Personen erfassen und für empirische Analysen zugänglich machen. Individuelle Bewegungen in Form von GPS-Daten objektiv zu erfassen, ist seit einigen Jahren über moderne Ortungsdienste auf Smartphones problemlos möglich. So konnten zum Beispiel Elgethun, Yost, Fitzpatrick et al. (2007) durch eine komparative Studie aufzeigen, dass die Bewegungsprofile von Kindern mittels GPS-Tracking wesentlich genauer erfasst werden können als durch Tagebuchzeitpläne von Seiten der Eltern. Wu, Jiang, Jaimes et al. (2013) betonen ebenfalls die Vorteile einer GPS-Erfassung im Vergleich zu subjektiven Einschätzungen. In einer Studie über das Reiseverhalten von Schwangeren konnten sie nachweisen, dass die Frauen tatsächlich wesentlich mehr Zeit im Straßenverkehr verbrachten und somit einer intensiveren Luftverschmutzung ausgesetzt waren, als dies die befragten Frauen selbst eingeschätzt hatten. Die Vorteile einer Methodenkombination von GPS-Daten, Fragebogenerhebung und qualitativen Textanalysen konnten auch Weber und Bauder (2013) zeigen. Zur Analyse von touristischen Praktiken verteilten sie unter ihren Probanden GPS-Geräte und Fragebögen, um einen Zusammenhang zwischen touristischen Typen (Fragebogen) und den tatsächlich besuchten Orten herzustellen. Mittels qualitativer Textanalyse, die sie aus Reiseführern und Ortsbeschreibungen entnommen haben, erhielten sie zusätzliche Informationen über die von den Touristen besuchten Orte und konnten diese so besser klassifizieren. Hier zeigen sich auf methodischer Ebene große Gemeinsamkeiten zu unserem Forschungsdesign. Der große Vorteil unseres Ansatzes besteht allerdings darin, dass wir die Sammlung der GPS-Daten und der Informationen über den Fragebogen innerhalb einer Anwendung (Smartphone-App) organisieren konnten.

\section{Methode}

Das Forschungsprojekt, an dem wir diese Vorüberlegungen veranschaulichen wollen, verfolgt drei Teilfragen: (1) Lassen sich studentische Bewegungsprofile entlang soziokultureller Merkmale beschreiben? (2) Lassen sich städtische Orte lokalisieren, die sich in Bezug auf soziokulturelle Merkmale ihrer Nutzer durch eine besonders heterogene oder besonders homogene Struktur auszeichnen? (3) Durch welche inhaltlichen Merkmale zeichnen sich die jeweiligen Orte als erfahrbare Qualitäten aus?

Während die ersten beiden Fragen auf die Klärung der soziokulturellen Differenzierung der Bewegungsmuster abzielen, zielt die dritte Frage auf eine inhaltliche Klärung der Hintergründe dafür, dass bestimmte Orte von bestimmten Gruppen aufgesucht werden, während andere Orte von anderen Gruppen aufgesucht werden und wieder andere keine solche selektive Nutzungsstruktur aufweisen. Während sich die ersten Fragen also auf strukturell-äußerliche Phänomene beziehen, betrifft die dritte Frage gerade die inhaltlichen Bedeutungsaspekte der Bewegungsmuster und Räume. Entsprechend dieser Zweiteilung des Erkenntnisinteresses soll hier auch eine Zweiteilung des methodischen Vorgehens umgesetzt werden: in quantitative Methoden einerseits und qualitative andererseits. Verfolgt wird demnach ein „Mixed-Methods-Ansatz". 


\subsection{Mixed Methods}

Allgemein unterscheiden Schoonenboom und Johnson (2017: 114) zwei Möglichkeiten der quantitativ-qualitativen Methodenkombination: "concurrent/indenpendent" und „sequential/dependent“. Während im ersten Verfahren qualitative und quantitative Methoden unabhängig voneinander einen Forschungsgegenstand betrachten, besteht im zweiten Verfahren ein Abhängigkeitsverhältnis zwischen den Ansätzen, indem die Ergebnisse der Analyse des einen Methodenansatzes als Orientierung für den zweiten dienen.

Mayring (2001) unterscheidet zwischen verschiedenen Formen sequenzieller Forschungsdesigns. Die sogenannten Vorstudien stellen „eine klassische Variante quantitativ orientierten Vorgehens" dar. Hier werden qualitative Verfahren verwendet, um Hypothesen für eine quantitative Hauptstudie zu gewinnen. Forschungsdesigns im Sinne eines Verallgemeinerungsmodells sehen ebenfalls qualitative Verfahren als Vorstufe für quantitative Verfahren an, erstere erhalten hier jedoch einen höheren Stellenwert. Ergebnisse der qualitativen Studien werden nach diesem Design durch quantitative Verfahren abgesichert bzw. verallgemeinert (vgl. Mayring 2001). Gemäß des Vertiefungsmodells, und das ist für die vorliegende Studie von Belang, werden die Ergebnisse der quantitativen Studie durch qualitative Analysen erweitert bzw. vertieft. Dieses Modell entspricht auch dem von Schooneboom und Johnson (2017: 117) vorgeschlagenen Verfahren des explanatory sequential designs. Nach diesem Verfahren folgt einer ersten Analyse und Auswertung quantitativer Daten eine qualitative Erhebung und Auswertung, die zu einem besseren Verständnis der quantitativen Ergebnisse beitragen soll. Burzan (2016: 40 f.) differenziert dieses Verfahren noch einmal nach der Funktion der qualitativen (Nach-) Studie. Die qualitative Studie kann (a) als eine illustrierende Bestätigung der quantitativen Befunde verwendet werden, (b) als Ergänzung und Verfeinerung oder (c) als Aufklärung unklarer Befunde.

Für die vorliegende Fragestellung bietet sich das explanatory sequential design nach Schoneboom und Johnson (2017) an, mit dem Ziel einer illustrierenden Bestätigung ${ }^{3}$ im Sinne Burzans (2016). Einer ersten Analyse und Auswertung quantitativer Daten

3 Für das Forschungsdesign insgesamt bieten sich alle drei der von Burzan (2016) genannten Typen einer Vertiefungsstudie an. So ist auch vorstellbar, dass sich widersprechende Ergebnisse mithilfe qualitativer Beobachtungen oder Interviews untersuchen lassen. Dies war aber nicht Ziel des vorliegenden Forschungsinteresses. folgt demnach eine qualitative Erhebung und Auswertung, die zu einem besseren Verständnis und zu einer Illustration der quantitativen Ergebnisse beitragen soll. Entsprechend diesem Vorgehen haben wir zunächst mit einer Smartphone-Applikation GPS-Daten von Probanden gesammelt. Zusätzlich erhielt jeder Proband eine Push-Nachricht mit einem Link zu einem externen Onlinefragebogen, der in erster Linie soziodemographische und Lebensstilmerkmale erfassen sollte. Mit dieser Kombination konnten wir die Bewegungsmuster der Probanden entlang soziokultureller Kriterien beschreiben (Forschungsfrage 1). Zusätzlich bestand so aber auch die Möglichkeit, Orte zu identifizieren, die in Bezug auf Lebensstilmerkmale eine sehr heterogene oder eine sehr homogene Nutzungsstruktur aufwiesen (Forschungsfrage 2). Die so identifizierten Orte wurden dann in einem weiteren Analyseschritt besucht und anhand qualitativer Erhebungsmethoden beschrieben (Forschungsfrage 3). Der zunächst rein deskriptiv-quantitativen Lokalisation folgte also eine inhaltlich-qualitative Interpretation. Konzeptionell folgt unser Mixed-Methods-Ansatz der Idee des qualitativen GIS-Ansatzes ${ }^{4}$, der vorwiegend innerhalb humangeographischer Forschungsarbeiten eingesetzt und diskutiert wird (Bittner/Michel 2018: 152). Der qualitative GIS-Ansatz verbindet physisch-geographische Daten mit qualitativem Forschungsmaterial, welches in Form von Beobachtungen, Interviews, Text-, Ton-, Bild- und Videoanalysen gewonnen wurde. Diese Methoden- und Materialkombination kann dann eingesetzt werden, um die geographischen Daten soziokulturell zu kontextualisieren (Schäfer/Muenchow/Harnisch 2018: 168). „Dieses Zusammenführen von Wissensformen und Ergebnisse [sic!] unterschiedlichster Disziplinen ist Grundlage der sogenannten kombinierten Forschungsmethoden (mixed methods) und gleichzeitig integraler Bestandteil qualitativer GIS-Forschung" (Schäfer/Muenchow/Harnisch 2018: 169). In dieser Kombination sehen wir ein enormes Potenzial für die Bearbeitung vieler stadtsoziologischer und stadtplanerischer Fragestellungen.

\subsection{Verfahren zur Erhebung und Auswertung der Bewegungsprofile}

Die verwendete Software zur Erfassung der Bewegungsprofile und soziokulturellen Merkmale bestand aus drei Komponenten: (1) einer Applikation (App) für Mobilfunkgeräte (bisher nur für solche mit Android-Betriebssys-

4 Geographische Informationssysteme. 
tem) zur anonymisierten Erfassung der geographischen Daten, (2) einer Benachrichtigungsfunktion, mit der den Probanden eine Push-Nachricht zugesandt werden konnte, sowie (3) einem Server als Endpunkt zum Empfangen, Weiterverarbeiten und Speichern der Daten in einer Datenbank und einem Interface zum Zugriff auf die Datenbank.

Für die Entwicklung der App wurde auf Dienste von Google Play zurückgegriffen. Sie sollte in regelmäßigen Intervallen die Standortinformationen der Probanden erfassen, speichern und an den externen Server senden. Jede der von den mobilen Clients erfasste Positionsangabe enthielt dabei Informationen über Längengrad, Breitengrad, Geschwindigkeit, Höhe über Null, Genauigkeit, Kurs und einen Zeitstempel. Zusätzlich wurde den Probanden eine Push-Nachricht zugesendet, die einen Link zu einem externen Fragebogen enthielt. Dieser beinhaltete neben soziodemographischen Fragen hauptsächlich Fragen nach dem Lebensstil der Studierenden. Eine Option, Lebensstile sowohl knapp als auch präzise zu erfragen, bietet die Typologie von Otte (2004). Otte verfolgte bei der Konstruktion dieser Typologie zwei übergeordnete Ziele: Er strebte (1) eine Typologie an, deren Typenbildung nicht a posteriori mittels mathematischer Zuweisungsverfahren (z. B. Cluster- oder Korrespondenzanalysen) vorgenommen wird, sondern a priori entlang „etablierter, theoretisierter und empirisch zentraler Dimensionen der Lebensführung" (Otte 2005: 444). Zudem wollte er (2) Lebensführungstypen vorlegen, die sich mithilfe weniger Indikatoren operationalisieren lassen, damit "sich die Typologie in Bevölkerungsumfragen unaufwändig replizieren lässt“" (Otte 2005: 444). Ziel ist es demnach auch, Lebensführung als handhabbare, vielfach verwendete Globalvariable zu etablieren (vgl. Otte 2011: 362). Gerade die Möglichkeit der Operationalisierung von Lebensführung mit nur zehn Items war für unsere Zwecke entscheidend. Aufgrund der hohen Sensibilität der gesammelten Bewegungsdaten und den Schwierigkeiten der Akquirierung von Versuchsteilnehmern wollten wir kein zusätzliches Risiko für unit-noneresponses ${ }^{5}$ eingehen, die mit einem überproportional aufgeblähten Fragebogen womöglich hätten provoziert werden können.

Alle Daten wurden auf einen externen Server übertragen. Auf dem mobilen Client (Smartphone) wurden die Daten in einer SQLite-Datenbank zwischengespeichert und bei bestehender Internetverbindung als JSONObjekt an den Back-End-API-Server übertragen, wo sie

5 Unit-non-response: Nichtbeantwortung des kompletten Fragebogens. analysiert, überprüft und dauerhaft in der PostgreSQLDatenbank gespeichert werden konnten. Anhand von Identifikationsnummern, die Limesurvey als sogenannte Tokens (Merkmale) zur Verfügung stellt, konnten die einzelnen Bewegungsdatensätze mit den soziokulturellen und Lebensstilmerkmalen verknüpft und entsprechend ausgewertet werden.

\subsection{Verfahren zur Erhebung und Auswertung der qualitativen Beobachtungen}

Anhaltspunkte zur Beobachtung der lokalisierten Orte waren gruppenspezifische Platzierungen von Gegenständen und symbolische Grenzziehungen. Zur empirischen Erhebung von solchen Platzierungen oder Raumgestaltungselementen orientieren wir uns an Herbert Schuberts Konzept der Soziologischen MehrebenenArchitektur-Analyse (Schubert 2005: 13 f.). Da für unsere Fragestellung lediglich die architektonische Gestaltung von Innenräumen in ihrer Wirkung auf die sozialen Nutzermilieus relevant ist, beschränken wir uns im Folgenden auf zwei Ebenen von Schuberts Konzept (vgl. Tabelle 1).

Auf der sozialen Ebene betrachtet Schubert die Korrespondenz der baulichen Gestalt mit den sozialen Figurationen der Nutzer (Schubert 2005: 17 f.) sowie die individuelle Zivilisierung, die danach fragt, wie gebaute Umwelt das Verhalten von Personen beeinflusst (Schubert 2005: 18). Während wir Ersteres hauptsächlich über materielle Merkmale des Raums operationalisiert haben, bezog sich die zweite Dimension auf den Einfluss der Gestaltungselemente auf individuelle Verhaltensmöglichkeiten. So beobachteten wir beispielsweise die Art und Weise, wie Steh- und Sitzgelegenheiten verteilt sind und wie diese bestimmte Bewegungsmöglichkeiten der Nutzer einschränken oder fördern. Auf der symbolischen Ebene ist für unser Forschungsziel Schuberts Dimension der Raumkultur relevant. Bei der Erfassung der Raumkultur geht es um semantische Codes der alltäglichen Raumnutzung. Hier fragten wir beispielsweise nach den Dekorationsgegenständen, nach Gemälden, Kunstdrucken, Postern, Werbeschildern oder Wandmalereien, die den Einrichtungsstil der jeweiligen Lokalität prägen.

\subsection{Exkurs: Datenschutz}

Spätestens seit den Aufdeckungen von Edward Snowden und den Skandalen um Cambridge Analytica 
Tabelle 1: Beobachtungskonzept in Anlehnung an Schubert (2005)

\begin{tabular}{|c|c|c|}
\hline \multicolumn{3}{|l|}{ 1. Soziale Ebene } \\
\hline \multirow{8}{*}{$\begin{array}{l}\text { Korrespondenz mit sozialer Figuration der } \\
\text { Nutzer }\end{array}$} & \multirow{5}{*}{$\begin{array}{l}\text { Informationen über den } \\
\text { Raum }\end{array}$} & Größe (grobe Einschätzung) \\
\hline & & Raumaufteilung \\
\hline & & Deckenhöhe \\
\hline & & Etagenzahl \\
\hline & & Raucherbereich \\
\hline & \multirow{3}{*}{$\begin{array}{l}\text { Informationen über die } \\
\text { Raumnutzung }\end{array}$} & Wie bewegen sich die Personen im Raum? \\
\hline & & Gibt es Schwellen der Raumnutzung? \\
\hline & & Wie werden die Personen im Raum organisiert? \\
\hline \multirow{7}{*}{$\begin{array}{l}\text { Individuelle Zivilisierung (architektonische } \\
\text { Elemente, die das Verhalten beeinflussen) }\end{array}$} & \multirow{4}{*}{ Beleuchtung } & Lichtverhältnisse \\
\hline & & Organisation von Lampen \\
\hline & & Kerzen \\
\hline & & Fenster (z. B. offen einsehbar, verdeckt, klein) \\
\hline & \multirow[t]{2}{*}{ Sitzplatzverteilung } & Tische \\
\hline & & Personen/Tisch \\
\hline & Einrichtungsstil & Subjektive Beschreibung der Einrichtung \\
\hline \multirow[t]{3}{*}{ Verhalten der Personen } & & Expressiv vs. zurückhaltend \\
\hline & & Laut vs. leise (Zimmerlautstärke) \\
\hline & & Statische vs. dynamische Raumnutzung \\
\hline \multicolumn{3}{|l|}{ 2. Symbolische Ebene } \\
\hline \multirow[t]{3}{*}{ Raumkultur } & \multirow[t]{3}{*}{ Semantische Codes } & Dekorationsgegenstände \\
\hline & & $\begin{array}{l}\text { Wandgestaltung (Kunstwerke, Kunstdrucke, } \\
\text { Photographien, Wandmalereien, Werbeschilder etc.) }\end{array}$ \\
\hline & & Einrichtungsstil \\
\hline
\end{tabular}

und die diversen Sicherheitslücken bei Facebook ist die Erhebung personengebundener Daten ein sensibles Thema. Insbesondere Standortdaten können viele, durchaus intime Details des Privatlebens preisgeben. Die Erhebung und Auswertung von GPS-Daten erfordert daher eine gewisse Vorsicht und entsprechende Kommunikation und weitgehende Aufklärung der Teilnehmenden. Als zentrales Kommunikationsmedium diente uns eine eigens eingerichtete Homepage, auf der zum einen ein Link zur App platziert war, zum anderen aber auch wichtige Informationen zum Ziel des Forschungsprojektes und zur Art und Weise des Umgangs mit den erhobenen Daten abgerufen werden konnten. Auf diese Weise informierten wir die Studierenden darüber, dass wir die Daten lediglich zu Forschungszwecken verwenden, sie nur auf den Servern der Universität gespeichert werden und eine Weitergabe an Dritte ausgeschlossen ist. Ferner wurden sie auch darüber informiert, welche Informationen wir im Einzelnen mit der App erheben und wer auf diese Zugriff haben würde.

\section{Ergebnisse}

Die von uns durchgeführte Erhebung fand im Sommersemester 2017 an der Universität Koblenz-Landau am Campus Koblenz statt. Als Teilnehmende an der Erhebung konnten rund 60 Koblenzer Studierende gewonnen werden, welche die von uns entwickelte App für einen Zeitraum von zwei Wochen auf ihrem Mobiltelefon installierten und aktivierten. Über diesen begrenzten Zeitraum hinweg konnten wir ihre Bewegungsdaten sammeln und auswerten. Aufgrund der geringen Fallzahl und einiger technischer Schwierigkeiten der verwende- 

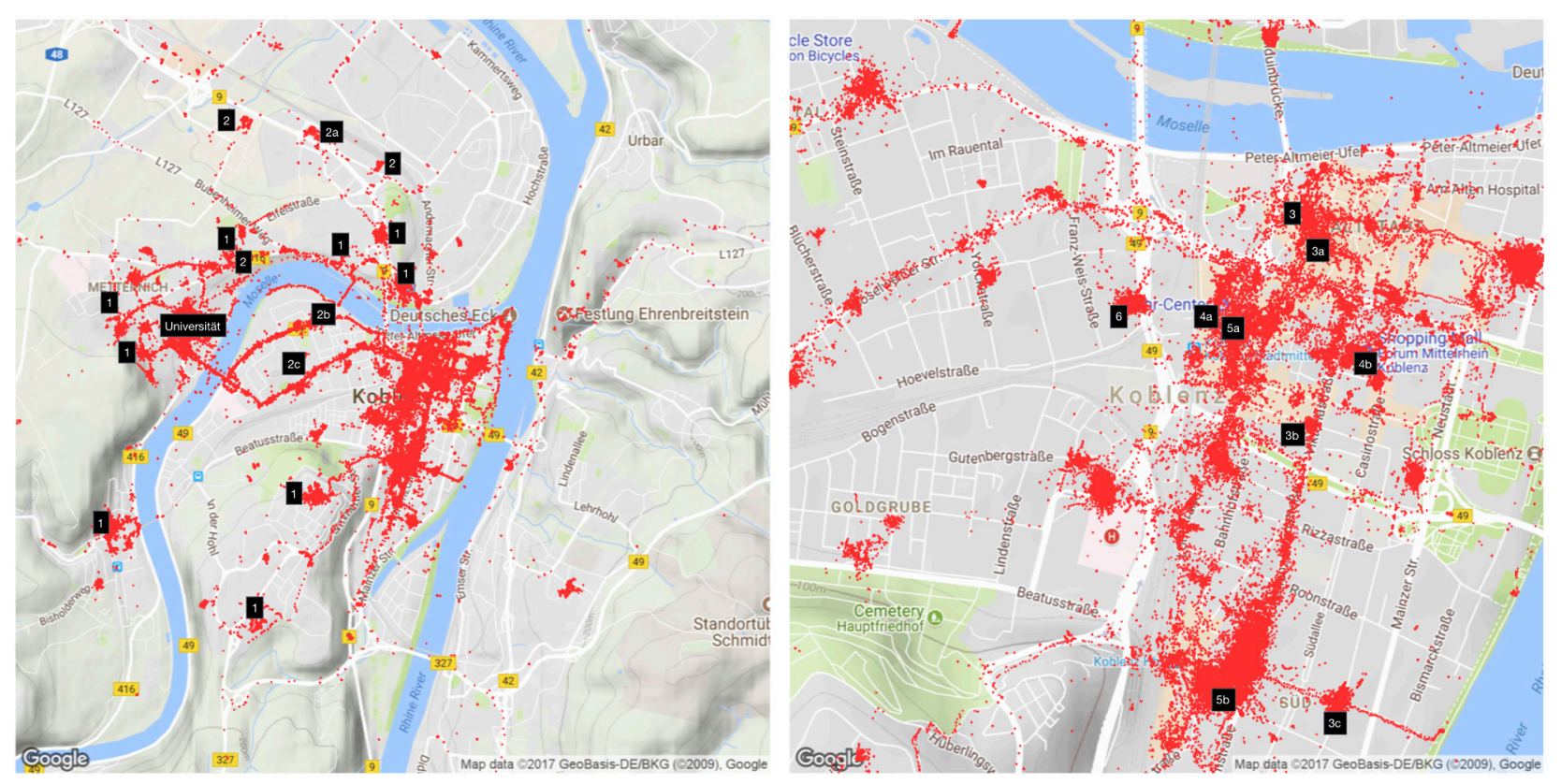

Abbildung 1: Die am häufigsten von Studierenden frequentierten Orte (Hotspots). Erläuterung: 1 = Wohngebiete; 2 = Einkaufsmöglichkeiten $(2 \mathrm{a}=$ Edeka, $2 \mathrm{~b}=$ Hit-Markt, $2 \mathrm{c}=$ Aldi); $3=$ Restaurants, Bars und Kneipen; 4 = Einkaufszentren; $5=$ Verkehrsknotenpunkte; $6=$ Fitnessstudio

ten App erheben wir keinen Anspruch auf Repräsentativität der Ergebnisse. Das Projekt sollte eher als eine Studie der prinzipiellen Umsetzbarkeit des Konzeptes und der möglichen Potenziale des Ansatzes angesehen werden. Dementsprechend erachten wir unser Projekt schon dadurch als erfolgreich, dass Bewegungsdaten erfolgreich aufgezeichnet und gespeichert, mit den ausgefüllten Fragebögen verknüpft und statistisch und geographisch ausgewertet werden konnten.

\subsection{Die am häufigesten frequentierten Orte (Hotspots)}

Abbildung 1 zeigt eine erste Übersicht über die Bewegungsdaten der Probanden. ${ }^{6}$ Bereits auf dieser Stufe der Auswertung lassen sich zentrale Orte (Hotspots) identifizieren, die von den Teilnehmern häufig besucht wurden.

Neben dem Campus selbst, der der mit Abstand am meisten frequentierte Ort ist, lassen sich durch basale Ortskenntnis bereits Wohngebiete (1), Einkaufsmöglichkeiten (2) und diverse Orte der Freizeitgestaltung (3,

6 Die Auswertungen wurden mit der Analysesoftware "R“ durchgeführt. Für die Darstellung wurde das R-Paket "ggmap“ verwendet und als Datenquelle für die Kartendarstellung das entsprechende Angebot von Google.
4, 6) differenzieren. Die identifizierten Wohnstandorte der Studierenden befinden sich entweder in näherer Umgebung zur Universität oder im Stadtteil Lützel, der als eines der günstigeren Wohngebiete gilt, und dementsprechend bei den in der Regel noch relativ einkommensschwachen Studenten äußerst beliebt ist. Weitere Wohnstandorte lassen sich aber auch in den Stadtteilen Karthause und Pfaffendorf lokalisieren.

Bei den identifizierten Einkaufsmöglichkeiten (2) handelt es sich vor allem um Supermärkte oder Discounter. Die hier markierten Geschäfte liegen gerade nicht im Zentrum, sondern in den wohnstandortnahen Gebieten außerhalb der Innenstadt. Ähnliche Angebote oder Gelegenheiten finden sich auch in der Innenstadt.

Der rechte Teil der Abbildung zeigt einen vergrößerten Ausschnitt der Koblenzer Innenstadt. Südlich an die Mosel angrenzend befindet sich die Koblenzer Altstadt mit großem Gastronomieangebot und einigen Boutiquen, weiter im Süden folgt die Stadtmitte, in der neben Gastronomie auch zahlreiche Einkaufsmöglichkeiten vorrangig für Güter des regelmäßigen Bedarfs vorhanden sind. In der Umgebung des Hauptbahnhofs beginnt die südliche Vorstadt, die sich als Wohnort wachsender Beliebtheit erfreut. Zwischen Stadtmitte und Südstadt sind noch einige speziellere Einzelhändler und Gastronomieangebote anzutreffen, zudem verschiedene 
Behörden sowie die einzigen noch im Betrieb befindlichen Kinos der Stadt.

Die hier identifizierbaren Hotspots lassen sich differenzieren in die Kategorien "Restaurant, Bar und Kneipe“ (3), die zwei großen ECE-Einkaufszentren „Löhr-Center“ und „Forum Mittelrhein“ (4) und in Verkehrsknotenpunkte und Umsteigeplätze wie den Koblenzer Hauptbahnhof (5b) oder den Busbahnhof vor dem Löhr-Center (5a). Einen letzten, hier nur exemplarisch angeführten Hotspot stellt ein bekanntes Fitnessstudio dar (6).

Eine erste Erkenntnis, die sich aus dieser undifferenzierten Abbildung bereits ableiten lässt, ist, dass die Bewegungsprofile rund um den jeweiligen Ort der Wohnung eine sehr hohe Konzentration aufweisen. Dies legt die Vermutung nahe, dass die Studierenden sich in ihrem Wohnquartier vorrangig in ihrer eigenen Wohnung aufhalten und die direkte Umgebung kaum nutzen. Dies trifft sowohl auf die Wohngebiete im Stadtteil Metternich (westlich der Universität) als auch auf die in Lützel (nördlich der Mosel) zu. Die Bewegungsprofile jenseits von Universität und Wohnort konzentrieren sich hingegen mehrheitlich auf den Innenstadtbereich, der, vor allem in der Altstadt, zahlreiche Ausgehlokalitäten wie etwa Restaurants, Bars, Kneipen und Clubs aufweist. Diese Diffusion der Aktionsräume der Studierenden weg von ihrem Wohngebiet hin zu Ausgehmöglichkeiten kann als Indiz für einen Wandel in der Beziehung von Milieu und Raum, wie inn Schulze (1994) beschreibt, gelesen werden. Nach Schulze ist die räumliche Zugehörigkeit eines Menschen als äußerliches Attribut immer weniger evident und zur Kategorisierung desselben immer weniger brauchbar (Schulze 1994: 44 f.). Anstelle der früheren Bedeutung von Raum als Umgebung, die für die Angehörigen eines Milieus als gemeinsamer Lebensund Identifikationsraum beansprucht wird, wird Raum nach Schulze mehr und mehr als Szenerie genutzt, das heißt als bewusst gewählter, zeitlich begrenzter Treffpunkt, der als Bühne zur Selbstdarstellung der Milieuangehörigen fungiert (Schulze 1994: 46). Insofern sich unsere lebensweltlichen Überzeugungen und Identitäten in der alläglichen Interaktion mit unserer sozialen Umwelt ausbilden, entspricht es dieser Interpretation, wenn die Aktionsräume sich weniger im Wohngebiet (Raum als Umgebung) als in den verschiedenen Ausgehmöglichkeiten verteilen (Raum als Szenerien). Interessant sind hier auch Überlegungen, die Martina Löw in ihrer Raumsoziologie anstößt. Nach ihr gibt es nicht ein vorherrschendes, ausschließliches Verhältnis zwischen Milieu und Raum, sondern die verschiedenen Milieus konstituieren den Raum auf je eigene Weise. „Milieus arbeitsloser Emigranten zum Beispiel konstituieren
Raum anders als Milieus der ,young urban professionals', soziologisch interessant ist das differente Wie der Konstitution" (Löw 2001: 257). Raum kann demnach, je nach Blickwinkel, am selben Ort zugleich als Umgebung und als Szenerie konstituiert werden. Um diese Überlegungen zu überprüfen, wäre allerdings ein breiterer Vergleich nötig, als inn die doch noch relativ homogene Gruppe der Studierenden erlaubt.

\subsection{Bewegungsprofile nach Lebensstiltypen}

Das Erkenntnispotenzial der Bewegungsprofile kann erhöht werden, wenn diese nach der Lebensführungstypologie differenziert werden (vgl. Abbildung 2). Aus Gründen der Fallzahlen werden hier nur die Typen „Hedonisten“ (grün) und „Aufstiegsorientierte“ (rot) dargestellt. Nach Otte zeichnen sich die „Hedonisten“ durch die Handlungslogiken „Jugendkultureller Stilprotest durch Mode und Musik, Innovationsfreude, gegenwartsbezogene Genuss- und Konsumorientierung, Extraversion, städtische Spektakel- und Clubkultur" (Otte 2005: 454) aus. Die „Aufstiegsorientierten“ sind hingegen durch die Handlungslogiken „Zentriertheit um solide Berufskarriere, Familie und Partizipation am Mainstream der modernen Freizeitkultur, ,Durchschnittlichkeit' und interne Heterogenität des Typus durch Mittelposition“ (Otte 2005: 454) charakterisiert.

Ein erster Überblick zeigt eine relative Dominanz der „Aufstiegsorientierten“ in den Gebieten südlich der Altstadt, während die „Hedonisten" häufiger bestimmte Orte im Altstadtbereich aufsuchen. Betrachtet man den Altstadtbereich etwas genauer, so findet sich nur ein Ort (5), der deutlich von den „Aufstiegsorientierten“ dominiert wird. Bei diesem handelt es sich um eine Filiale der Gastronomiekette „Extrablatt“, die sich durch ein breites Spektrum angebotener Speisen und Getränke auszeichnet. Für die „Hedonisten“ Iassen sich insbesondere zwei Lokalitäten im Westen der Altstadt identifizieren, die sich dem Bereich der Szenekneipen zuordnen lassen. Dabei handelt es sich um das "Excalibur" (1) und das „Fox“ (4). Entlang unserer Beobachtungskategorien ist es nun möglich, diese Lokalitäten näher zu beschreiben und mit den Lebensstiltypen zu vergleichen. Für eine bessere Kontrastierung zwischen den zwei Lebensstiltypen beschränken wir uns im Folgenden auf die Beschreibung der Lokalitäten „Fox“ (Hedonisten) und „Extrablatt“ (Aufstiegsorientierte).

Das „Fox“ ist eine kleine bis mittelgroße Kneipe in der Koblenzer Altstadt. Der Gastraum wird von einer 

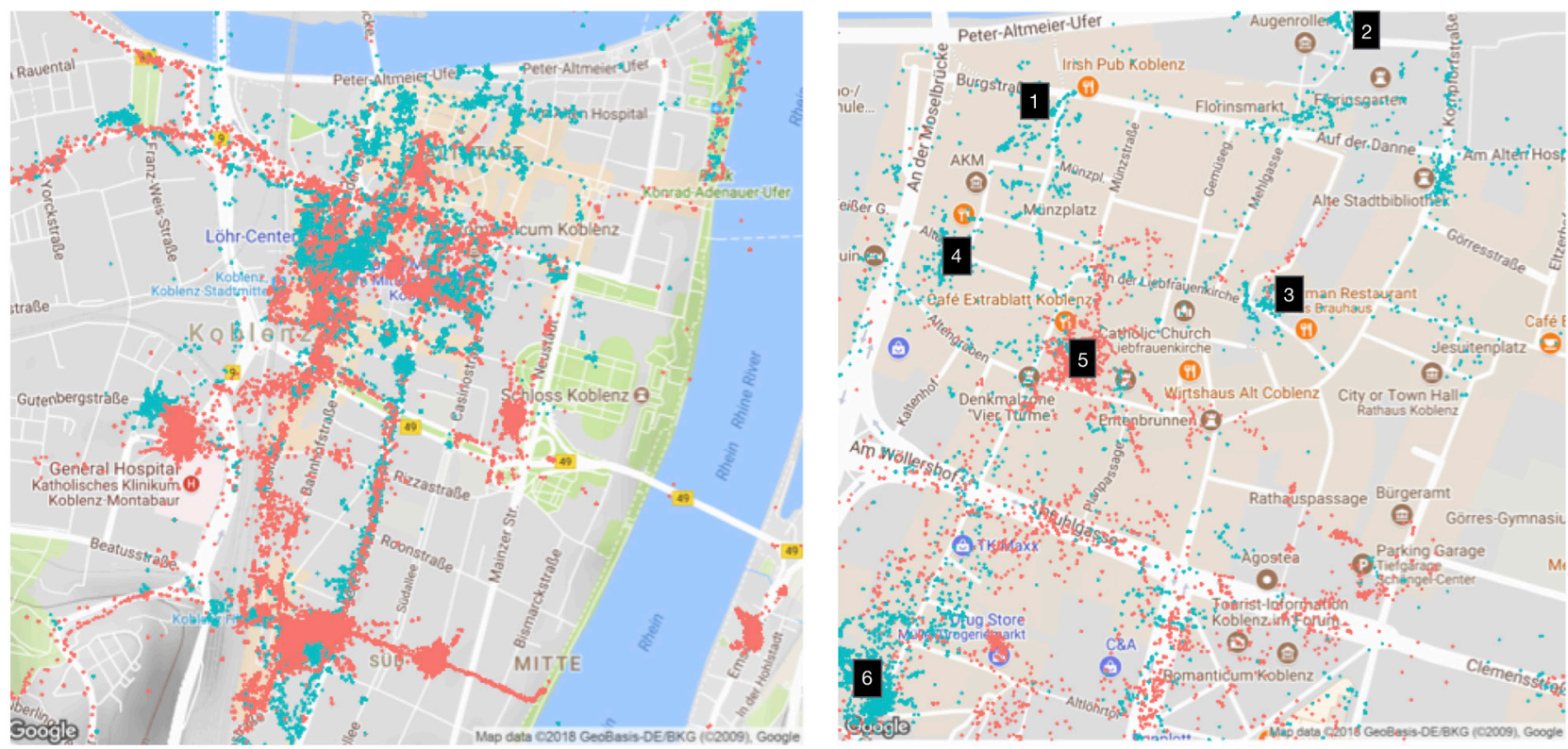

Abbildung 2: Bewegungsprofile nach Lebensführungstypen: Hedonisten (grün), Aufstiegsorientierte (rot). Erläuterung: $1=$ Excalibur (Rockkneipe); 2 = Cocktailbar; 3 = (beliebteste) Eisdiele; 4 = Fox (Tanzbar); 5 = Extrablatt (Restaurant, Café, Bar); $6=$ REWE To Go

großen Theke in der Mitte dominiert, um die sich die übrigen Einrichtungsgegenstände verteilen. Die nur wenigen Sitzgelegenheiten sind entlang mehrerer Tische, die fast eine längere Tafel bilden, angeordnet. In der Mitte des Raumes, direkt vor der Theke, ist viel Platz, auf dem gestanden und auch getanzt werden kann. An der linken Seite des Raumes befindet sich ein DJ-Pult. Die Fenster an der Vorderseite bestehen aus Butzenglas und sind von außen nur bedingt einsehbar. Der Innenraum ist somit deutlich gegen die Außenwelt abgegrenzt. Die Beleuchtung besteht aus Schirmlampen, Lichterketten, bunten Strahlern, einer Diskokugel und leuchtenden Werbeschildern. Trotz der vielen Lichtquellen ist es relativ dunkel im Raum. Der Großteil der Besucher befindet sich auf der Tanzfläche, was aufgrund der Anordnung der Einrichtungsgegenstände als durchaus intendiert erscheint. Zwischendurch setzt man sich in wechselnden Gruppen auf die Sitzgelegenheiten, steht aber bald wieder auf, geht an die Theke, tanzt oder setzt sich an einen anderen Tisch. Die Raumnutzung ist daher sehr dynamisch. Die Wände sind mit verschiedenen Bemalungen und Bildern verziert. Die altmodischen Schirmlampen erscheinen in diesem Kontext eher als ,Retro'-Stilelement denn als altbacken. Insgesamt scheint die Raumkultur an die Lebenswelt des Publikums angepasst: Es handelt sich durchweg um Inhalte und Gestaltungselemente einer studentischen Populärkultur. Alles ist bunt, neonfarben, schrill, extravagant. Die Musik ist gemischt, laut und steht deutlich im Vordergrund. Insgesamt zielt die Raumkonzeption auf exzessives Feiern. Durch Betreten der Bar soll man die Außenwelt hinter sich lassen und sich frei fühlen für neue Erfahrungen, neue Bekanntschaften, überbordende Gefühlswelten.

Das „Extrablatt“ bietet einen deutlichen Kontrast zum „Fox“. Der Gastraum ist groß, hell und weit; er erstreckt sich über zwei Ebenen mit einer Galerie. Die lange Theke ist an den Rand gerückt und drängt nicht ins Zentrum. Auf beiden Seiten gibt es große Fensterfronten, sodass es kaum eine Sitzgelegenheit gibt, die nicht von außen einsehbar wäre. Es gibt sehr modern aussehende Deckenleuchter und Wandbeleuchtungen. Wenn es draußen dunkel ist, ist der Gastraum hell erleuchtet. Das Licht ist sehr warm, sodass eine angenehm freundliche Atmosphäre entsteht. Die Sitzgelegenheiten sind um kleinere und größere Tische gruppiert, die sich gleichmäßig über die zwei Etagen verteilen. Die Gäste sitzen in geschlossenen kleineren oder größeren Gruppen oder auch nur zu zweit an ihren Tischen, speisen, trinken Cocktails und unterhalten sich. Die Einrichtung ist solide, gepflegt und übersichtlich. Die Tische bestehen aus dunklem Holz, die Wände sind beige und mit Kunstfotographien, gerahmten Bildern oder Werbeschildern verziert. Der Einrichtungsstil folgt dem Ideal der Art déco. Alles wirkt gedeckt, zurückhaltend, aber auch modern und urban. Angesprochen wird ein breites Publikum, das weite Teile der Mittelschicht aus Stadt und 
Umland umfassen dürfte. Jeder fühlt sich von dem einen oder anderen Gestaltungselement angesprochen und, was noch wichtiger ist, keiner fühlt sich von irgendetwas unangenehm überrascht oder berührt. Alles ist angenehm, beruhigend und, gewohnt'. Auch im Tagesablauf versucht das "Extrablatt" flexibel auf eine Vielzahl von Bedürfnissen zu reagieren. Morgens bis mittags kann man hier frühstücken bzw. brunchen. Mittags bis abends gibt es Snacks und größere Speisen. Will man das kulinarische Angebot des „Extrablatt" begrifflich verdichten, so bietet sich die Bezeichnung des "kulturellen“ oder, in leichter Abwandlung, des "kulinarischen Synkretismus“ an (Gebhardt 2010: 301). Das Angebot reicht von italienischen, amerikanischen, englischen Frühstücksmenüs bis zu ebenso breiten Angeboten für Mittag- oder Abendessen. $^{7}$

Nach dieser Charakterisierung scheint das „Extrablatt“ in der Tat dem Typus „aufstiegsorientiert" in Ottes Lebensführungstypologie zu entsprechen. Dieser ist an "Durchschnittlichkeit" orientiert und weist eine "interne Heterogenität [...] durch Mittelposition" auf (Otte 2005: 454). Dieser Nichtfestgelegtheit auf profilierte Lebenswelten entspricht in vielerlei Hinsicht der „kulturelle Synkretismus“, den wir im „Extrablatt“ identifiziert haben. Sie zeigt sich darüber hinaus auch deutlich in der Raumgestaltung, die nach Möglichkeit völlig auf extravagante, szenetypische oder gar avantgardistische Symboliken, die eine exkludierende Wirkung haben könnten, verzichtet. Das „Fox“ hingegen entspricht merklich dem Typus der „Hedonisten“, die Otte (2005: 454) unter anderem durch „Extraversion" und „städtische Spektakel- und Clubkultur" charakterisiert. Hier wird offensiv ein bestimmter Lebensentwurf ausgelebt und dargestellt. Es wird sich demonstrativ abgegrenzt vom Mainstream und exzessiv gefeiert. Am Beispiel beider Lokalitäten zeigt sich somit der von Klee (2001) formulierte „Raumbezug von Lebensstilen“. Denn sowohl das „Fox" als auch das „Extrablatt“ können als lebensstilbezogene gastronomische Einrichtungen im Sinne Klees (2001: 76) verstanden werden.

Neben diesen soziokulturell ,homogenen' Ausgehlokalitäten konnten wir auch vergleichsweise ,heterogene' Orte identifizieren. Dabei handelt es sich vor allem um die bereits erwähnten Einkaufszentren, die mehr oder weniger gleichmäßig von den verschiedenen Lebensführungstypen aufgesucht wurden. Ohne hier tiefer auf die Implikationen der soziokulturellen Heterogenität eingehen zu können, sei zumindest erwähnt, dass solche Lokalitäten mehr noch als eines „kulturel-

7 https://cafe-extrablatt.de/speisekarte/ (29.01.2019). len Synkretismus" einer weitreichenden Milieuneutralität bedürfen (Schulze 1994: 46), welche sich auch als „Milieuuniversalismus" verstehen ließe. Anders als beim „Fox“ und mehr noch als beim „Extrablatt" müssen die Innenraumgestalter der beiden Einkaufszentren darauf achten, keine milieuspezifischen Symbole zu platzieren, welche zum Ausschluss bestimmter Gruppen potenzieller Kunden führen könnten. Auch diese intendierte Breite der Kundschaft lässt sich mit unserer Methode abbilden.

\section{Fazit}

Auch in Anbetracht der Tatsache, dass unsere inhaltlichen Ergebnisse und Interpretationen keinerlei Anspruch auf Repräsentativität beanspruchen können, lassen sich bereits aus der Untersuchung einige Schlussfolgerungen ableiten. Durch die Erneuerung und Modernisierung der Aktionsraumforschung konnten wir zeigen, dass eine Rekonstruktion von Bewegungsprofilen oder eben Aktionsräumen vermittelst einer App möglich ist. Dementsprechend identifizierten wir zunächst Hotspots, also insgesamt besonders häufig frequentierte Orte. Über die Fragebogenerhebung war es uns möglich, diese Orte nach Lebensführungsmerkmalen zu differenzieren. So konnten wir zum einen soziokulturell sehr homogene Orte als milieuspezifische Treffpunkte herauszufiltern. Gerade diese Treffpunkte leisten auch der Interpretation Gerhard Schulzes Vorschub, dass das Verhältnis der Milieus zum Raum sich - zumindest für die von uns erfassten Studierenden - vermehrt als "Szenerie" darstellt (Schulze 1994). Darüber hinaus konnten wir neben diesen soziokulturell homogenen Orten andererseits auch soziokulturell sehr heterogene Orte ausfindig machen. Bei aller Vorsicht der Interpretation zeigen aber gerade die homogenen Orte - als die Lokalisation milieuspezifischer Raumbezüge - das große Potenzial unseres Forschungsdesigns. Bereits mit den vorhandenen Daten konnten wir Anzeichen einer engen Passung von theoretisch implizierten Annahmen zu den Lebensführungstypen und den Raumbezügen einerseits und den aufgesuchten Lokalitäten andererseits feststellen.

Dieses Forschungsdesign bietet sich für eine Vielzahl von Fragen innerhalb der Stadtforschung an. So wäre ein naheliegendes Anwendungsgebiet die Analyse urbaner Räume und gruppenspezifischer Aktionsräume entlang sozialstruktureller Kriterien (wie z. B. Bildung, Beruf, Einkommen). Außerdem wäre die Ausweitung der Untersuchungspopulation auf die gesamte Stadtbevölkerung interessant. Hier zeigt sich allerdings eine Grenze der Methodik, denn sie ist nur in den Teilen der 
Bevölkerung anwendbar, die im Besitz eines Smartphones sind. Eine Beschränkung auf die Untersuchung bestimmter Altersgruppen scheint daher vorerst schwer umgänglich.

Neben allgemeinen Analysen gruppenbezogener Bewegungsprofile lassen sich auch speziellere Fragestellungen in Bezug auf Raumaneignungs- und ErschlieBungsprozesse erforschen. Auf der Seite des Stadtraums lassen sich Klassifikationen von Gelegenheitsstrukturen erstellen, die aus milieuspezifischen Treffpunkten bestehen und sich unter Umständen über den gesamten städtischen Raum verteilen, das heißt, quer zu abgrenzbaren Raumeinheiten liegen. In leichter Abwandlung Parks (1952) ließe sich hier von „moral locations“ (statt von „moral regions") sprechen. An die Identifikation der Treffpunkte könnten sich qualitative Forschungsfragen anschließen, die ergänzend zum hier vorgestellten Ansatz anhand von qualitativen Befragungen der Personen ,vor Ort' nach Gründen forschen, warum sie diese und keine andere Gelegenheit bevorzugt besuchen. Aus derartigen Untersuchungen ließen sich dann möglicherweise Indikatoren ableiten, die bei der Gestaltung öffentlicher Räume als Räume der Begegnung berücksichtigt werden können. Solche öffentlichen Räume, in denen soziale Gegensätze sichtbar zu Tage treten, „offer opportunities to build up a sense of solidarity" (Shaftoe 2008: 13). Denn aus der Konfrontation mit gesellschaftlicher Heterogenität kann nach Klamt (2012: 786) „anonymisiertes, ,blasiertes', aber eben (anders gewendet) auch tolerantes Stadtleben resultieren“. Da solche Indikatoren induktiv auf der Basis von Beobachtungen und Befragungen der Personen, also der Stadtbevölkerung, gewonnen werden, können stadtplanerische Konzepte im Sinne eines Bottom-up-Prozesses entwickelt werden. Mit anderen Worten: Die Art und Weise, wie die Räume von der Stadtbevölkerung genutzt werden, sind für die Gestaltung handlungsleitend. Denn „[b]ei der Betrachtung öffentlicher Räume ist [...] kein Kriterium so entscheidend wie die Perspektive der Nutzer, denn sie ,lesen', erkennen, machen einen Raum zu einem ,öffentlichen Raum“ - oder eben nicht“" (Klamt 2012: 778).

\section{Literatur}

Allport, G. W. (1971): Die Natur des Vorurteils. Köln.

Bittner, C.; Michel, B. (2018): Qualitative Geographische Informationssysteme. Kontextsensible räumliche Analysen, mixed methods und Geovisualisierungen. In: Wintzer, J. (Hrsg.): Sozialraum erforschen: Qualitative Methoden in der Geographie. Berlin, 151-166. doi: 10.1007/978-3-662-56277-2
Blasius, J.; Friedrichs, J.; Symann, S. (2007): Armut und Lebensführung in einem benachteiligten Wohngebiet Kölns. In: Baum, D. (Hrsg.): Die Stadt in der Sozialen Arbeit. Ein Handbuch für soziale und planende Berufe. Wiesbaden, 188203. doi: 10.1007/978-3-531-90725-3

Blasius, J.; Winkler, J. (1989): Gibt es die „feinen Unterschiede“? Eine empirische Überprüfung der Bourdieuschen Theorie. In: Kölner Zeitschrift für Soziologie und Sozialpsychologie 41, 1, 72-94.

Bourdieu, P. (1982): Die feinen Unterschiede. Kritik der gesellschaftlichen Urteilskraft. Frankfurt am Main.

Burzan, N. (2016): Methodenplurale Forschung. Chancen und Probleme von Mixed Methods. Weinheim/Basel.

Cutler, D. M.; Glaeser, E. L.; Vigdor, J. L. (1999): The Rise and Decline of the American Ghetto. In: Journal of Political Economy 107, 3, 455-506. doi: 10.1086/250069

Dangschat, J. S. (2014): Residentielle Segregation. In: Gans, P. (Hrsg.): Räumliche Auswirkungen der internationalen Migration. Hannover, 63-77. = Forschungsberichte der ARL 3.

Dangschat, J. S.; Droth, W.; Friedrichs, J.; Kiehl, K. (1982): Aktionsräume von Stadtbewohnern. Eine empirische Untersuchung in der Region Hamburg. Opladen. = Beiträge zur sozialwissenschaftlichen Forschung 36.

Eder Sandtner, S.; Schneider-Sliwa, R. (2007): Neue Gesellschaftsformen und ihre residenziellen Verteilungsmuster am Beispiel von Basel-Stadt. In: Dangschat, J. S.; Hamedinger, A. (Hrsg.): Lebensstile, soziale Lagen und Siedlungsstrukturen. Hannover, 139-160. = Forschungs- und Sitzungsberichte der ARL 230.

Elgethun, K.; Yost, M. G.; Fitzpatrick, C. T. E.; Nyerges, T. L.; Fenske, R. A. (2007): Comparison of global positioning system (GPS) tracking and parent-report diaries to characterize children's time-location patterns. In: Journal of Exposure Science and Environmental Epidemiology 17, 2, 196-206. doi: 10.1038/sj.jes.7500496

Erdmann, T. (2001): Überlagerung von sozialer und ethnischer Segregation in Hamburg. In: Europa Regional 9, 3, 146-151.

Farwick, A. (2001): Segregierte Armut in der Stadt. Ursachen und soziale Folgen der räumlichen Konzentration von Sozialhilfeempfängern. Opladen. = Stadt, Raum und Gesellschaft 14.

Farwick, A. (2012): Segregation. In: Eckardt, F. (Hrsg.): Handbuch Stadtsoziologie. Wiesbaden, 381-419. doi: 10.1007/978-3-53194112-7

Friedrichs, J. (1983): Stadtanalyse. Soziale und räumliche Organisation der Gesellschaft. Opladen.

Friedrichs, J. (1990): Aktionsräume von Stadtbewohnern verschiedener Lebensphasen. In: Bertels, L.; Herlyn, U. (Hrsg.): Lebenslauf und Raumerfahrung. Opladen, 161-178. = Biographie und Gesellschaft 9.

Friedrichs, J. (2011): Ist die Besonderheit des Städtischen auch die Besonderheit der Stadtsoziologie? In: Herrmann, H.; Keller, C.; Neef, R.; Ruhne, R. (Hrsg.): Die Besonderheit des Städtischen. Entwicklungslinien der Stadt(soziologie). Wiesbaden, 33-47. doi: 10.1007/978-3-531-93338-2

Friedrichs, J.; Triemer, S. (2009): Gespaltene Städte? Soziale und ethnische Segregation in deutschen Großstädten. Wiesbaden. doi: 10.1007/978-3-531-91675-0 
Gebhardt, W. (2010): Die Eventisierung der Kultur. Strategien der kulturellen Verdummung. In: Revue d'Allemagne et des Pays de langue allemande 42, 3, 291-306.

Häußermann, H.; Siebel, W. (2004): Stadtsoziologie. Eine Einführung. Frankfurt am Main/New York.

Helbig, M.; Jähnen, S. (2018): Wie brüchig ist die soziale Architektur unserer Städte? Trends und Analysen der Segregation in 74 deutschen Städten. Berlin. = Wissenschaftszentrum Berlin Discussion Paper P 2018-001.

Hesse, M. (2011): Aktionsraum. In: Reutlinger, C.; Fritsche, C.; Lingg, E. (Hrsg.): Raumwissenschaftliche Basics. Eine Einführung für die soziale Arbeit. Wiesbaden, 25-33. = Sozialraumforschung und Sozialraumarbeit 7. doi: 10.1007/978-3-531-92619-3

Horton, F. E.; Reynolds, D. R. (1971): Effects of Urban Spatial Structure on Individual Behavior. In: Economic Geography 47, 1, 36-48. doi: 10.2307/143224

ILS - Institut für Landes- und Stadtentwicklungsforschung; Strohmeier, K. P. (2003): Sozialraumanalyse - Soziale, ethnische und demografische Segregation in den nordrheinwestfälischen Städten. Gutachten für die Enquetekommission "Zukunft der Städte in NRW“ des Landtags NordrheinWestfalen. Dortmund/Bochum.

Klamt, M. (2012): Öffentliche Räume. In: Eckardt, F. (Hrsg.): Handbuch Stadtsoziologie. Wiesbaden, 775-804. doi: 10.1007/978-3-531-94112-7

Klee, A. (2001): Der Raumbezug von Lebensstilen in der Stadt. Ein Diskurs über eine schwierige Beziehung mit empirischen Befunden aus der Stadt Nürnberg. Passau. = Münchener Geographische Hefte 83.

Krämer-Badoni, T. (1992): Die Stadt als sozialwissenschaftlicher Gegenstand. In: Häußermann, H.; Ipsen, D.; Krämer-Badoni, T.; Läpple, D.; Rodenstein, M.; Siebel, W. (Hrsg.): Stadt und Raum. Soziologische Analysen. Pfaffenweiler, 1-29. = Stadt, Raum und Gesellschaft 1.

Kühl, J.; Wörmer, S. (2012): Aktionsräume in polyzentrischen Stadtregionen - Ein Abbild räumlicher Entgrenzung? In: Growe, A.; Heider, K.; Lamker, C.; Paßlick, S.; Terfrüchte, T. (Hrsg.): Polyzentrale Stadtregionen. Die Region als planerischer Handlungsraum. Hannover, 217-228. = Arbeitsberichte der ARL 3.

Löw, M. (2001): Raumsoziologie. Frankfurt am Main.

Mayring, P. (2001): Kombination und Integration qualitativer und quantitativer Analyse. In: Forum Qualitative Sozialforschung $2,1,6$.

Otte, G. (2004): Sozialstrukturanalysen mit Lebensstilen. Eine Studie zur theoretischen und methodischen Neuorientierung der Lebensstilforschung. Wiesbaden.

Otte, G. (2005): Entwicklung und Test einer integrativen Typologie der Lebensführung für die Bundesrepublik Deutschland. In: Zeitschrift für Soziologie 34, 6, 442-467.

Otte, G. (2011): Die Erklärungskraft von Lebensstil- und klassischen Sozialstrukturkonzepten. In: Rössel, J.; Otte, G. (Hrsg.): Lebensstilforschung. Wiesbaden, 361-398. = Kölner Zeitschrift für Soziologie und Sozialpsychologie Sonderheft 51.

Park, R. E. (1915): The City: Suggestions for the Investigation of Human Behavior in the City Environment. In: American Journal of Sociology 20, 5, 577-612.

Park, R. E. (1952): Sociology, Community and Society. In: Park, R. E. (Hrsg.): Human Communities. The City and Human
Ecology. Glencoe, Illinois, 178-209. = The Collected Papers of Robert Ezra Park 2.

Pettigrew, T. F.; Tropp, L. R. (2006): A Meta-Analytic Test of Intergroup Contact Theory. In: Journal of Personality and Social Psychology 90, 5, 751-783. doi: 10.1037/0022-3514.90.5.751

Reuband, K.-H. (2018): Das Kulturpublikum im städtischen Kontext. Wie sich das Publikum der Oper von anderen Kulturpublika unterscheidet. In: Reuband, K.-H. (Hrsg.): Oper, Publikum und Gesellschaft. Wiesbaden, 143-191. doi: 10.1007/978-3-65812926-2

Schäfer, S.; Muenchow, J.; Harnisch, B. (2018): Qualitative Forschung und Geographische Informationssysteme. In: Wintzer, J. (Hrsg.): Sozialraum erforschen: Qualitative Methoden in der Geographie. Berlin, 167-180. doi: 10.1007/9783-662-56277-2

Schneider, N.; Spellerberg, A. (1999): Lebensstile, Wohnbedürfnisse und räumliche Mobilität. Opladen.

Schoonenboom, J.; Johnson, R. B. (2017): How to construct a Mixed Methods Research Design. In: Baur, N.; Kelle, U.; Kuckartz, U. (Hrsg.): Mixed Methods. Wiesbaden, 107-131. = Kölner Zeitschrift für Soziologie und Sozialpsychologie, Sonderheft 57.

Schubert, H. (2005): Empirische Architektursoziologie. In: Die alte Stadt 32, 1, 1-27.

Schütz, M. W. (1985): Die Trennung von Jung und Alt in der Stadt. Eine vergleichende Analyse der Segregation von Altersgruppen in Hamburg und Wien. Hamburg. = Beiträge zur Stadtforschung 9.

Schulze, G. (1992): Die Erlebnisgesellschaft. Kultursoziologie der Gegenwart. Frankfurt am Main/New York.

Schulze, G. (1994): Milieu und Raum. In: Noller, P.; Prigge, W.; Ronneberger, K. (Hrsg.): Stadt-Welt. Über die Globalisierung städtischer Milieus. Frankfurt am Main/New York, 40-53. = Die Zukunft des Städtischen 6.

Sennett, R. (2001): Verfall und Ende des öffentlichen Lebens. Die Tyrannei der Intimität. Frankfurt am Main.

Sennett, R. (2009): Civitas. Die Großstadt und die Kultur des Unterschieds. Berlin.

Shaftoe, H. (2008): Convival Urban Spaces. Creating Effective Public Places. London.

Siebel, W. (2012): Stadt und soziale Ungleichheit. In: Leviathan. Berliner Zeitschrift für Sozialwissenschaft. 40, 3, 462-475.

Vester, M. (2015): Die Grundmuster der alltäglichen Lebensführung und der Alltagskultur der sozialen Milieus. In: Freericks, R.; Brinkmann, D. (Hrsg.): Handbuch Freizeitsoziologie. Wiesbaden, 143-187. doi: 10.1007/978-3-658-01520-6

Weber, H.-J. L.; Bauder, M. (2013): Neue Methoden der Mobilitätsanalyse. Die Verbindung von GPS-Tracking mit quantitativen und qualitativen Methoden im Kontext des Tourismus. In: Raumforschung und Raumordnung 71, 2, 99-113. doi: 10.1007/s13147-013-0218-y

Wu, J.; Jiang, C.; Jaimes, G.; Bartell, S.; Dang, A.; Baker, D.; Delfino, R. J. (2013): Travel patterns during pregnancy: comparison between Global Positioning System (GPS) tracking and questionnaire data. In: Environmental Health 12, 86. doi: 10.1186/1476-069X-12-86 